\title{
Gradhiva
}

GRADHIV

Revue d'anthropologie et d'histoire des arts

15 | 2012

Robots étrangement humains

\section{Machines, magie, miracles}

Machines, magic, miracles

\section{Charles Malamoud}

\section{OpenEdition}

Journals

Édition électronique

URL : http://journals.openedition.org/gradhiva/2381

DOI : 10.4000/gradhiva.2381

ISSN : 1760-849X

\section{Éditeur}

Musée du quai Branly Jacques Chirac

\section{Édition imprimée}

Date de publication : 16 mai 2012

Pagination : 144-161

ISBN : 978-2-35744-047-0

ISSN : 0764-8928

Référence électronique

Charles Malamoud, « Machines, magie, miracles », Gradhiva [En ligne], 15 | 2012, mis en ligne le 16 mai 2015, consulté le 30 avril 2019. URL : http://journals.openedition.org/gradhiva/2381 ; DOI : 10.4000/ gradhiva.2381 







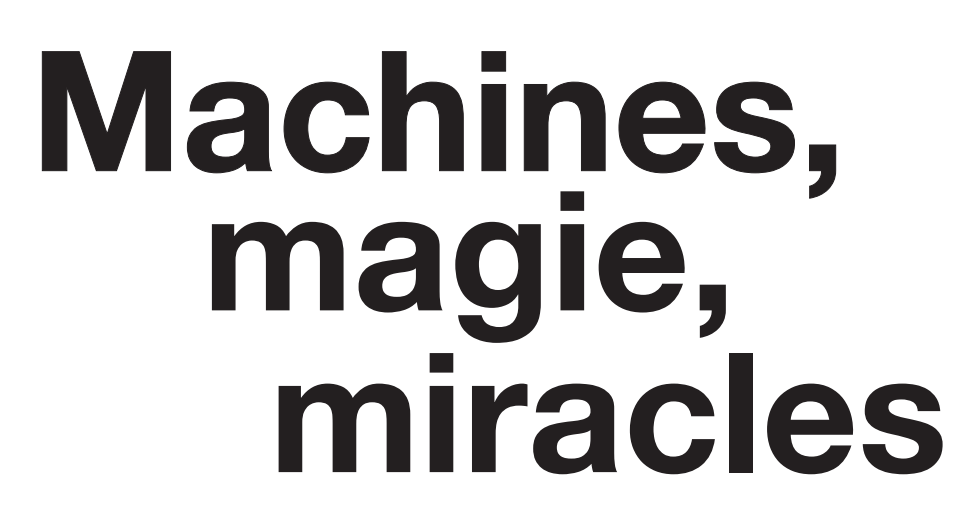

Charles Malamoud

Fig. 1 Abu'lzz Isma'il al-Jazari, Le Livre des connaissances : "L'horloge éléphant » @ The Metropolitan Museum of Art, Dist. RMN/image of the MMA.
Quelques mots du vocabulaire sanscrit sont familiers au locuteur français ou du moins pourraient l'être : ils sont enregistrés dans les dictionnaires d'usage, par exemple dans Le Petit Larousse illustré de 2007. Ce sont notamment (à côté des fameux karma et yoga) : mantra, sûtra, tantra. Ces noms à suffixe -tra sont des noms d'instruments : ils désignent ce à l'aide de quoi se réalise l'action exprimée par la racine verbale; ou encore ce en quoi ou à l'occasion de quoi cette action s'effectue. C'est ainsi que mantra signifie étymologiquement «instrument pour penser" (en fait, un mantra est un mot ou une brève séquence de mots censés être un support pour la méditation ou la réflexion; ce peut être aussi une formule qu'il faut réciter quand on exécute un geste rituel; ce peut être enfin l'avis, nettement formulé, que le roi reçoit de son conseiller, de son mantrin, terme qui est, lointainement, à l'origine du français «mandarin»). Un sûtra est proprement un «fil ", ce au moyen de quoi se réalise l'acte de «coudre "; ce mot s'emploie au sens de "fil directeur", aphorisme ou groupe d'aphorismes. Un tantra est d'abord une texture, ce dans quoi se réalise l'acte de tisser, et désigne le «texte».

Cette même série de noms en -tra comporte aussi un terme qui n'a pas encore acquis droit de cité en français et qui, cependant, est tout aussi important dans la civilisation de l'Inde traditionnelle que ceux que l'on vient d'énumérer: c'est yantra, dont le sens général est précisément «instrument» ou, mieux, "machine». Étymologiquement, le yantra est ce grâce à quoi on maintient, contient, retient une substance, une force, un mouvement (la racine verbale dont yantra est un dérivé signifie "maîtriser»). Ce peut être, dans sa forme la plus simple, une corde ou une barrière. Par exemple, dans le rituel de l'Inde la plus ancienne (de l'Inde que nous font connaître les textes du Veda), il faut poser à un certain emplacement de l'aire sacrificielle deux grosses tiges d'herbe ou deux cannes à sucre de telle manière qu'elles «séparent» deux zones, plus précisément deux strates de matière végétale qui couvrent ce terrain. Or on s'adresse 
à ces deux "séparateurs" avec ces mots : "Vous êtes deux yantra, deux barrières pour le peuple. " Barrières destinées à protéger, soutenir le peuple, la masse des sujets du roi, ou bien à le contenir dans les limites qui lui sont fixées ${ }^{1}$ ? Les deux sans doute. Dans cette même famille de textes (qui, d'une façon ou d'une autre, décrivent ou commentent les gestes du rite), le terme yantra désigne une structure destinée à étayer un objet et par là même à le maintenir en place. Il est dit que la terre qui menaçait, faut-il croire, d'errer ou de divaguer a été arrêtée ou stabilisée par le dieu Savitar au moyen de yantra (au pluriel), ce que Louis Renou traduit par "dispositifs freinants" ${ }^{2}$. Mais dans les textes plus tardifs, ceux qui appartiennent à l'Inde "classique", les yantra sont des instruments par lesquels on retient l'énergie pour pouvoir, en les relâchant brusquement, la libérer pour la diriger là où il faut. L'exemple le plus simple est celui de l'arc et de la flèche. Un exemple plus complexe mais de même type est la catapulte. Le terme yantra en vient à désigner toute espèce de dispositif permettant de mettre en mouvement (mais un mouvement toujours réglé et contrôlé) un objet : les rames et les voiles sont les yantra d'un bateau; de même, les cordes qui permettent de faire monter ou descendre un étendard le long d'un mât, la corde d'un puits, la roue à godets. On donne aussi le nom de yantra à cette machine mentale qu'est le diagramme : figure à deux dimensions, faite de traits et de points de couleurs convenues, qui permet de "visualiser " les cheminements de l'énergie cosmique telle qu'elle est conçue dans cette forme particulière des religions de l'Inde que l'on connaît en Occident sous le nom de «tantrisme».

Il y a des yantra plus mystérieux et plus puissants, qui appartiennent non pas à la vie prosaïque de tous les jours mais au monde fantastique des contes : ils sont conçus de manière à donner l'impression d'être des automates. Le vaste poème appelé Océan des rivières de contes (Kathâsaritsâgara), composé par Somadeva au $\mathrm{XI}^{\mathrm{e}}$ siècle de notre ère, contient au livre VII ${ }^{3}$ la description d'une ville très étrange :

Ils entrèrent dans la ville par l'artère commerçante. Là ils virent que tous les habitants, marchands, prostituées ou citoyens étaient d'étonnants automates de bois qui se mouvaient comme des êtres vivants, mais leur absence de langage montrait qu'ils étaient dépourvus de vie [...]. Les éléphants, les chevaux et autres créatures étaient faits de même [...]. À cet endroit, il aperçut un authentique être humain. Ce dernier était assis, entouré d'automates qui remplissaient les fonctions de portier ou de courtisane. Seul doué de conscience, il était cause du frémissement de ces objets inertes, les gouvernant comme l'âme gouverne les sens...

À ses visiteurs émerveillés, ce personnage, Râjyadhara, fait le récit de sa vie passée. Il leur parle du talent de charpentier qu'il avait en partage avec son frère, de leur habileté à produire des oies en bois qui, manœuvrées par des cordes agissant sur un système de chevilles, étaient capables de voler jusqu'au palais du roi, de pénétrer par un œil-de-bœuf dans la chambre aux trésors, de saisir des joyaux dans leur bec et de les rapporter à leur maître. Il leur conte aussi comment, craignant d'avoir été découvert, il a fui à bord d'un vaisseau volant construit de manière à pouvoir, propulsé par le vent, franchir en un instant des centaines de lieues (mais non toutefois de voler au-dessus des eaux). C'est à bord de ce véhicule aérien qu'il est arrivé jusqu'à cette ville déserte qu'il a peuplée d'automates et que ses visiteurs viennent de découvrir. Comprenons bien que ces machines si surprenantes par leurs performances sont mues par des mécanismes dissimulés mais en principe explicables, décomposables, sinon reproductibles: le talent, l'habileté technique de celui qui les a fabriquées sont hors du commun, ils ne sont pas à proprement surnaturels. Nous glissons cependant vers le surna- 


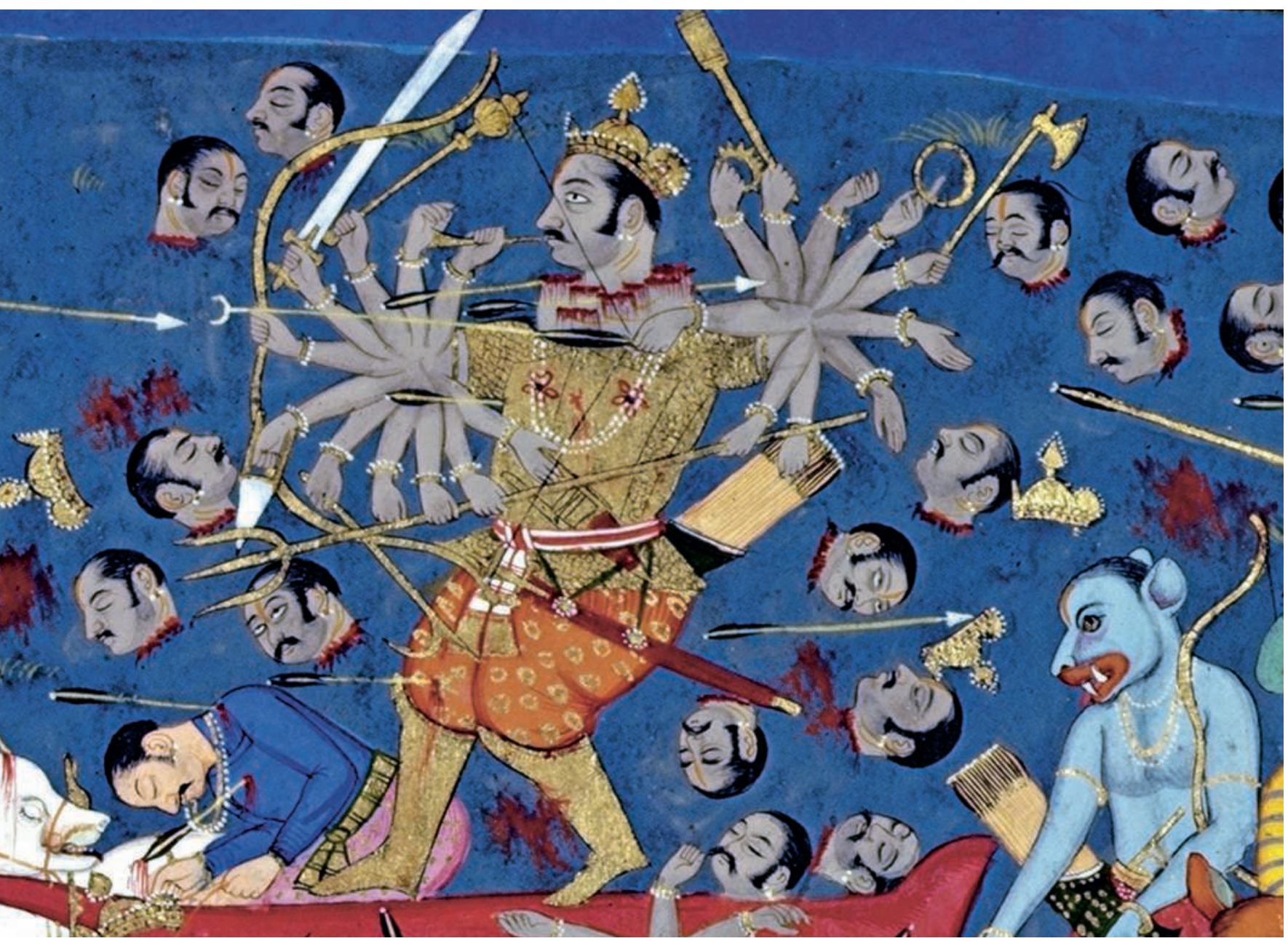

Fig. 2 Ravana perdant ses têtes, image du "Ramayana, Yuddha Kanda " publié pour la première fois à Udaipur, 1652. Détail @ akg-images/British Library.

turel quand celui qui règne sur ce peuple d'automates fait voir à ses hôtes qu'il lui suffit de penser à des mets pour qu'ils apparaissent devant lui, bien réels et prêts à être consommés par lui et par ses hôtes; en revanche, il a beau penser très intensément à des femmes, son ardeur imaginative ne parvient pas à les faire se matérialiser, il ne peut fabriquer ni susciter du vivant. Il arrive que ces véhicules volants et ces automates soient qualifiés de mâyâyantra, machines magiques, mais c'est pour amener une sorte de jeu de mots : elles sont comparées à celles que fabrique Maya, l'ingénieur et architecte divin.

La mâyâ proprement dite (ce nom est accueilli dans Le Petit Larousse) est autre chose que ce savoir-faire, cette habileté technique extraordinaire mais non nécessairement surnaturelle : c'est d'abord, chez certains dieux, le pouvoir de créer des formes, des formes changeantes, distinctes de la création cosmogonique proprement dite; chez le dieu Indra, c'est surtout le pouvoir de se transformer à volonté et d'échapper ainsi à ses adversaires. Puis c'est le pouvoir qu'ont les dieux, les démons et certains hommes de susciter des formes illusoires, des faux-semblants qui malgré leur caractère irréel ont la capacité de séduire ou d'épouvanter. Une idée très répandue et qui est au centre des spéculations de certaines écoles 
DOSSIER

ROBOTS ÉTRANGEMENT HUMAINS

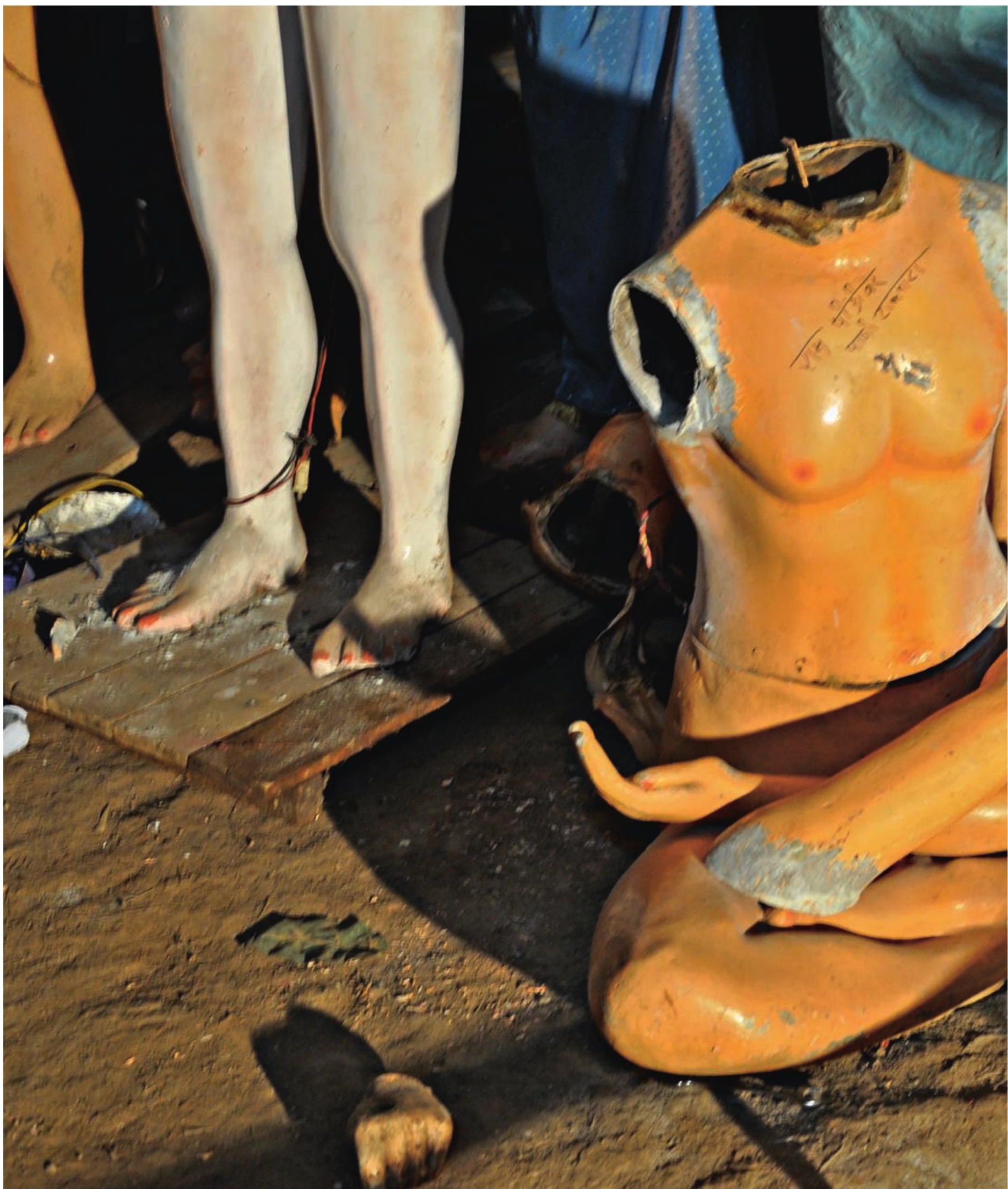




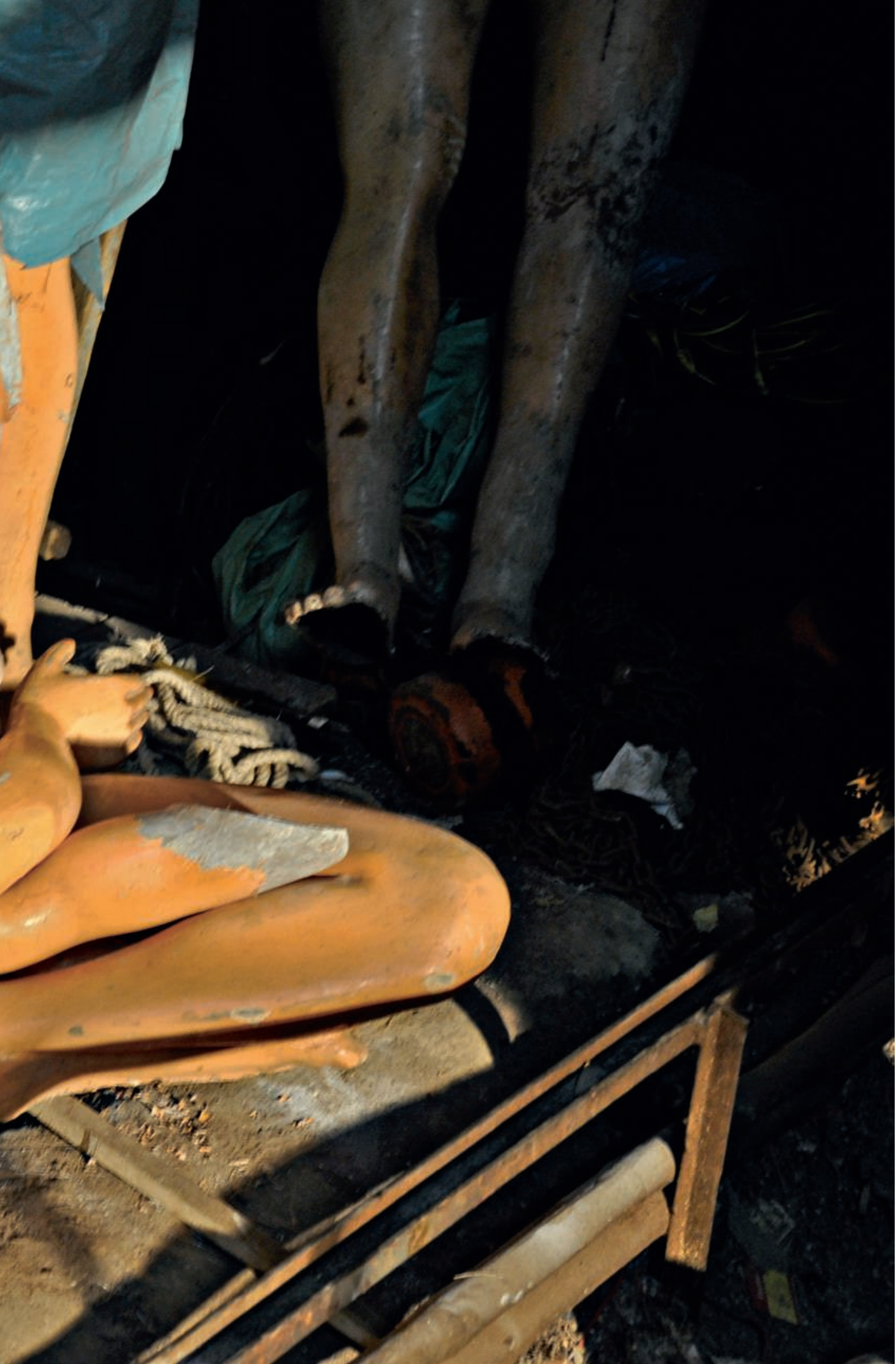

Fig. 3 Automate de sage hindou en position de yogi, prêt à être assemblé, Bombay, 2010. Photo Emmanuel Grimaud. 
de pensée est que la création elle-même est une illusion, un produit de la mâyâ divine : le monde tel qu'il nous apparaît dans sa diversité, avec ses objets multiples et limités, n'est qu'une apparence. Le texte le plus fameux de l'Inde, celui que tout le monde connaît et qu'on ne cesse de citer, la Bhagavad-Gîtâ, contient, en son chapitre final, cette strophe : «Le Seigneur, ô Arjuna, réside au cœur de tous les êtres, les faisant tournoyer par sa mâyâ comme s'ils étaient juchés sur un manège 4. . Les hommes ne sont pas seulement les spectateurs, ils sont aussi les acteurs, ou plutôt les marionnettes manipulées de cette fiction mise en spectacle qu'est le monde. Ce qui est rendu ici par «manège ", c'est yantra.

Parmi les hommes, il en est dont le métier est d'être des aindrajâlikavidyâkovida, illusionnistes et prestidigitateurs, littéralement « experts en la science des filets d'Indra " : leur savoir-faire est une mâyâ, ils usent d'accessoires qui sont des yantra de toute sorte. En ce sens, leur mâyâ n'est pas surnaturelle, elle est faite de trucs parfaitement démontables et ne fonctionne que parce que les hommes ont une propension à prendre les apparences pour la réalité, mais cela fait partie du bagout de ces artistes que de donner à leur art le nom de magie et aussi de se vanter des effets très réels que peut avoir sur la vie des spectateurs le fait de se laisser prendre au charme de l'apparence spectaculaire. Parmi ces auxiliaires matériels de l'illusion, il y a un collyre (anjana) dont on enduit les yeux des spectateurs pour les rendre plus réceptifs aux effets optiques, procédé comparable aux lunettes que l'on distribue aux spectateurs des films en 3D. Voici, extraite du roman picaresque intitulé Les Aventures des dix princes (de Dandin, viI siècle de notre ère), la description du spectacle donné à la cour du roi de Malava par un illusionniste particulièrement talentueux, le brahmane Vidyeshvara :

Tandis que résonnait le son des instruments frappés par les musiciens, que s'élevait, mélodieuse comme le chant des coucous ivres d'amour, la voix des chanteuses, dans un grand tournoiement de plumes de paon qui transporta de passion l'esprit des spectateurs, le magicien déploya ses partenaires en cercle, puis s'immobilisa un instant, les yeux clos. Alors surgirent des serpents qui vomissaient des flots de venin. Chaperon dressé, illuminant toute la façade du palais de l'éclat des joyaux incrustés sur leur tête, ils rampaient en jetant la terreur dans l'assistance. Puis des nuées de vautours s'abattirent sur ces reptiles monstrueux, les arrachèrent à coups de bec et les enlevèrent dans les cieux. Enfin, le brahmane fit paraître le dieu Narasimha en train d'étriper le démon Hiranyakashipu, mettant ainsi un comble à l'ébahissement du souverain.

Au pur divertissement se mêle une intrigue : le magicien inclut dans son spectacle et fait passer pour un simulacre la représentation d'un rite qui équivaut à un rite effectif, à savoir le mariage de la fille du roi avec son bien-aimé, un prince inconnu du roi et dont il était peu probable qu'il l'agréerait. Voici comment, à la faveur d'un faux-semblant, on crée un fait accompli :

"Seigneur, lui dit alors le magicien, il te faut conclure sur un spectacle faste. Pour t'assurer une prospérité éternelle, je vais célébrer le mariage d'une jeune personne qui est l'exacte réplique de ta fille avec un prince doté de tous les signes royaux.» Impatient d'admirer pareille cérémonie, le roi donna son assentiment. Le visage épanoui par la certitude de réussir son stratagème, le magicien instilla dans ses yeux un collyre générateur d'illusion pour toute l'assistance et promena ses regards autour de lui. Et, devant tous ces spectateurs convaincus d'assister à un tour de magie, il unit en mariage Râjavâhana débordant de passion avec Avantisundarî, qui parut alors, selon un plan convenu à l'avance, splendidement revêtue de ses plus beaux atours. Vidyeshvara, qui connaissait à la perfection rites et formules du mariage, fit apparaître le feu sacrificiel, témoin de cette union. Comme la cérémonie s'achevait, 


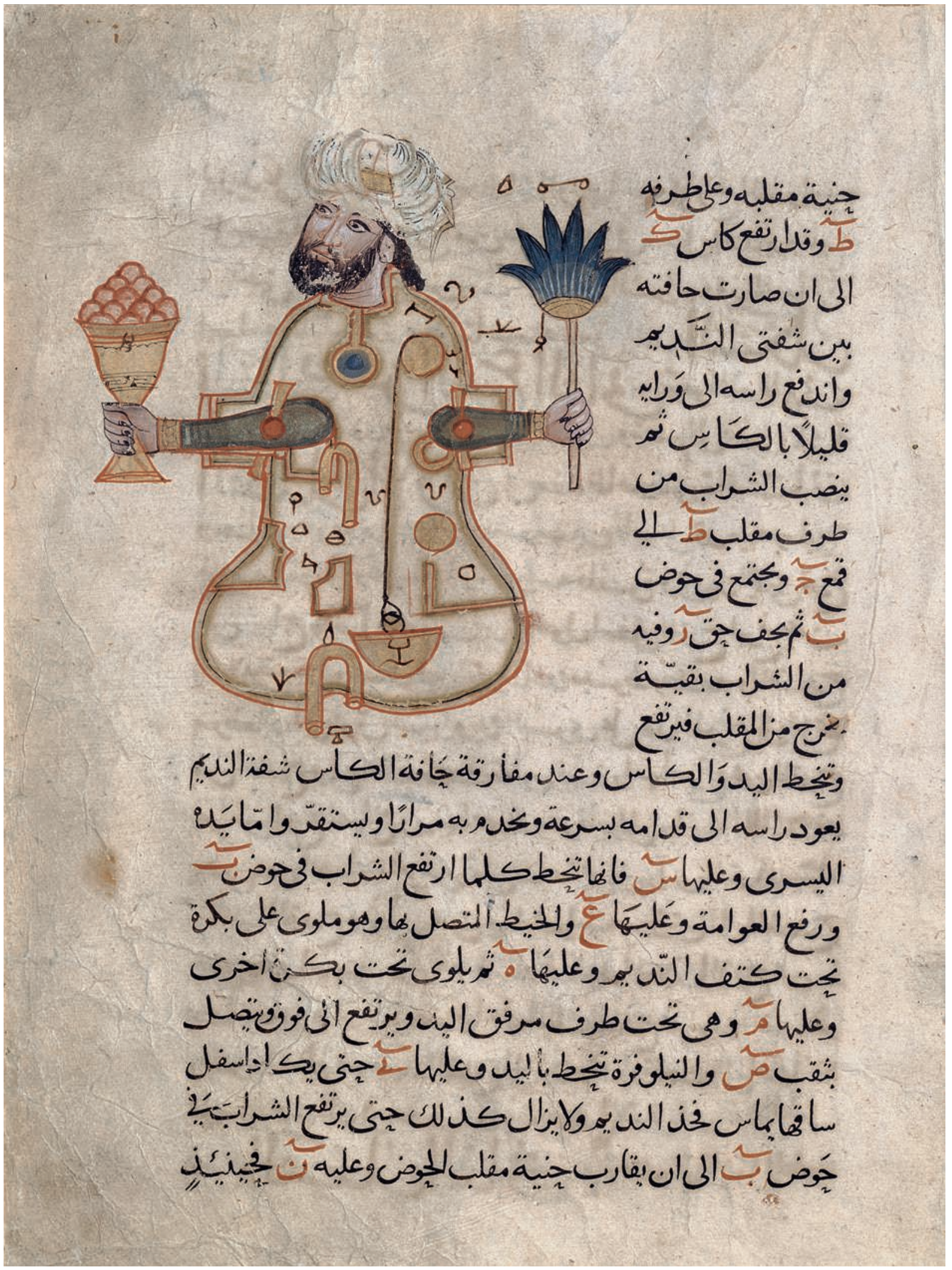

Fig. 4 «Figure pour l'utilisation des liquides à boire », Livre de la connaissance des procédés mécaniques par Abu'l Izz Isma'il al-Jazari; copié par : Farkh ibn Abd al-Latif, probablement syrien @ The Metropolitan Museum of Art, Dist. RMN-GP/image of the MMA. 


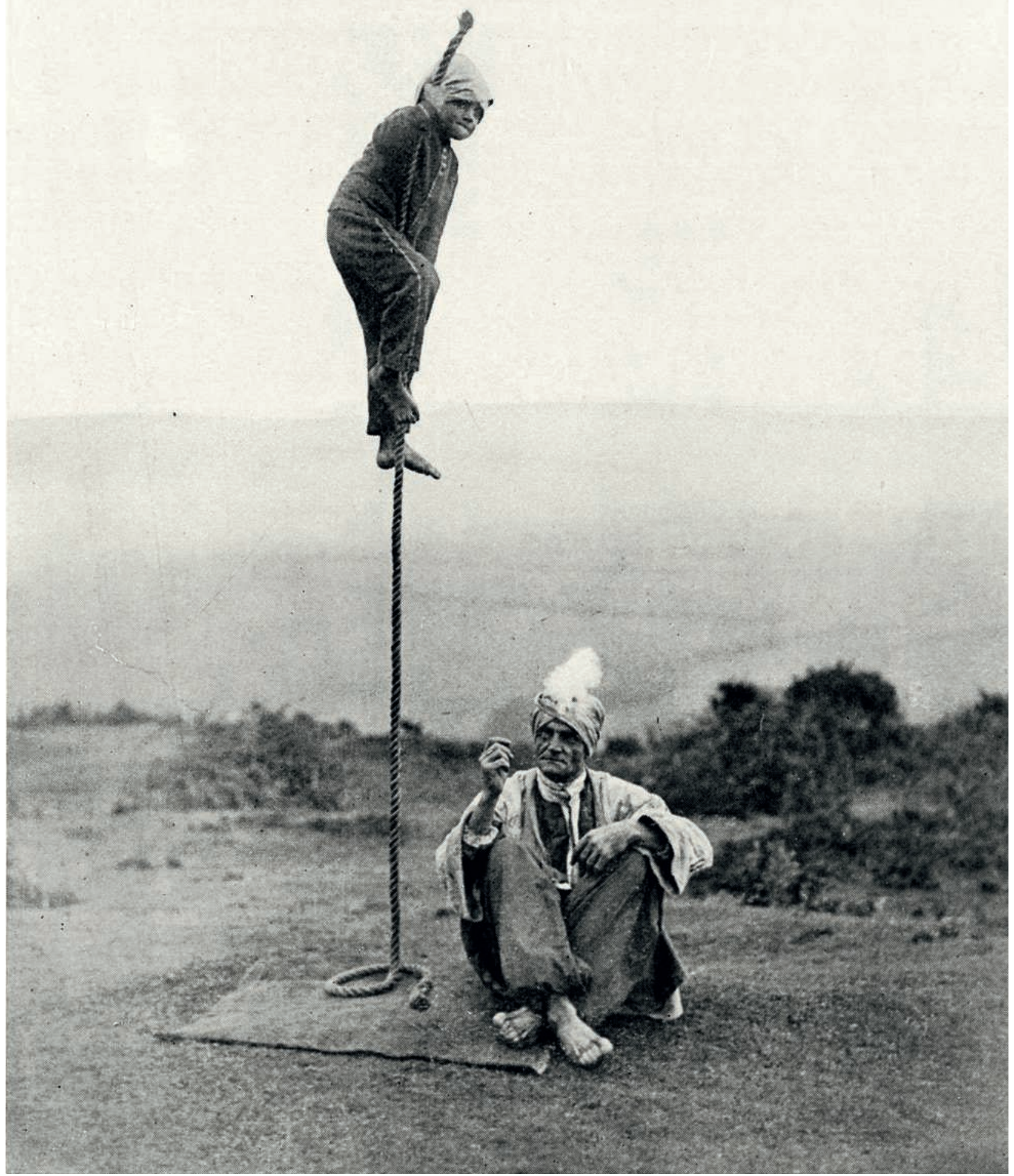

Fig. 5 Le tour indien de la corde exécuté par Karachi (de son vrai nom Arthur Derby), 1935. Price Collection/Senate House Library, University of London [le petit garçon est son fils]. 
le brahmane s'écria : "Créatures magiques, disparaissez!» et toutes ces figures illusoires s'évanouirent l'une après l'autre selon un plan mis au point à l'avance. Râjavâhana entra dans le gynécée comme s'il était lui-même un être de magie... Alors Avantisundarî, entourée de ses amies intimes, conduisit son époux bien-aimé dans une chambre somptueuse. Et Râjavâhana vit tous ses désirs comblés, par la force de la magie comme par la volonté des hommes ${ }^{5}$.

La relation entre yantra et mâyâ, machine et magie, est un thème essentiel du traité de « politique » de l'Inde ancienne, l'Arthaçâstra de Kautilya (ou Kautalya ${ }^{6}$ ). L'Arthaçâstra, littéralement "enseignement sur l'intérêt ", souvent comparé au Prince de Machiavel, expose ce que doit être le but du roi et les moyens dont il doit disposer pour l'atteindre.

Le but, c'est la conquête de la terre entière. Par définition, le roi est en permanence "désireux de conquête". Le roi auquel s'adresse l'auteur du traité est donc désigné ici comme "le roi conquérant" pour le distinguer des autres rois, ses alliés ou ses adversaires. Il doit préserver les territoires qu'il a acquis et constamment travailler à en gagner de nouveaux, ce qui correspond à peu près à la division entre politique intérieure et politique étrangère. Les moyens, ce sont les richesses de tout ordre qu'il peut tirer des territoires sur lesquels il règne. Les "éléments constitutifs" (prakrti) du royaume sont : le roi lui-même, le ministre, le pays, la place forte, le trésor, l'armée, l'allié. À chacune de ces instances correspond un secteur de l'" appareil d'État " (cf.VI 1). Il est remarquable que le " pays", janapada, littéralement le «sol des gens ", la terre porteuse d'une population, soit un élément parmi d'autres, un moyen parmi d'autres de la puissance royale. Les caractéristiques d'un bon "pays " sont énumérées en VI 1, 8 : il doit, notamment, être riche en ressources naturelles, ne pas dépendre exclusivement de la pluie pour son alimentation en eau, être capable de supporter amendes et impôts, être peuplé de paysans laborieux et appartenant pour la plupart à des castes inférieures, etc. L'armée et les forteresses ont dans leur équipement des yantra, des machines de guerre, qui sont en fait divers types de catapultes; on distingue notamment des yantra fixes et des yantra mobiles.

Ce qui n'apparaît pas dans cette énumération des prakrti, c'est que le roi dispose d'un immense réseau d'agents secrets, le plus souvent déguisés en ascètes ou saints hommes, qui le renseignent et lui permettent de monter toutes sortes de scénarios de provocation dans ce qu'on peut appeler la guerre psychologique. Cette police secrète est très diversifiée : il y a les agents à poste fixe et les itinérants; ceux qui sont spécialisés dans la collecte et la transmission d'informations et ceux qui sont chargés de mettre à mort les hommes dont le roi conquérant veut se débarrasser (ces agents sont les tîkshna, les "pointus " ou les "durs». On les classe aussi suivant le type de «couverture " qu'ils adoptent7). En revanche, il ne semble pas qu'il y ait une différence de statut ou de style entre les agents à l'intérieur du royaume et ceux qui agissent en territoire ennemi.

Un des objectifs tactiques du roi est de convaincre les populations, ses sujets comme ses adversaires, qu'il est omniscient et aussi qu'il est en communication directe avec les dieux, qu'il est leur familier et leur ami et que par conséquent il a des pouvoirs surnaturels. La guerre psychologique n'exclut pas une action sur les corps et peut comporter des assassinats très réels, mais le but est avant tout de frapper les esprits : terroriser, séduire, paralyser l'adversaire, l'empêcher de raisonner en captant son attention par des images qui sont des faux-semblants et relèvent donc de la mâyâ. Souvent, la production de ces images demande une machinerie plus ou moins complexe, dont nous ne saisissons pas toujours
- -

5. Dandin, Daçakumâracarita, I 5. Cf. Porcher 1995 : 104-10?.

6. Pour la tradition indienne, Kautilya était le conseiller de l'empereur Candragupta, dont le règne a commencé en 322 avant J.-C. On s'accorde à penser que le texte de l'Arthaçâstra est en fait nettement plus tardif; il date peutêtre du ${ }^{\text {er }}$ siècle avant ou après le début de notre ère. Rappelons que ce texte s'était perdu; des manuscrits en ont été trouvés au début du $x x^{e}$ siècle : un nouveau champ s'est alors ouvert dans les études indiennes.

7. Sur cette question, voir Scharfe 1968 : 233-276. 


\section{$\bullet \bullet$}

8. L'《élimination des épines 》 est un euphémisme pour désigner la tâche de la police et de la justice. À l'《élimination des épines 》, c'est-à-dire à la détection et à la répression des activités illégales, est consacré tout le livre IV. Il faut distinguer ces «épines 》 des personnes en qui le roi voit une menace ou une gêne pour lui-même : il s'en débarrasse par le «châtiment silencieux» confié aux services secrets. On présente comme de dangereux comploteurs, ennemis du roi conquérant, de simples délinquants qu'on avait déjà sous la main.

9. Le texte ne permet pas de comprendre par quel procédé cet effet est obtenu. Quoi qu'il en soit, le roi conquérant veut montrer non seulement qu'il est dans la familiarité des dieux, mais encore qu'il a lui-même des pouvoirs surnaturels. le mode de fonctionnement. Cette machinerie est faite de yantra. Ces yantra ne sont pas des automates, mais leur but est tout de même de faire surgir des images qui doivent donner l'impression qu'il s'agit d'êtres réels et autonomes. En tant qu'elle consiste en manœuvres secrètes, la guerre psychologique est faite d'une multiplicité de yoga : le lecteur occidental ne s'y attend pas, mais tel est bien le sens de ce mot dans l'Arthaçâstra. Le chapitre in du livre XIII, que l'on va examiner ci-après, a pour titre yogavâmana, littéralement les procédés secrets, yoga, auxquels le roi conquérant doit recourir pour forcer la ville qu'il assiège à vomir, vâmana, le roi ennemi qui y réside. Ouand, au chapitre vi du livre X, Kautilya énumère les moyens de semer la panique chez l'ennemi, sur le champ de bataille ou dans l'ensemble du pays et de la population, les yantra sont mentionnés dans la même liste que les "pratiques secrètes ", les "durs » ou " pointus " (tîkshna) des services secrets et les mâyâ, « magies créatrices de faux-semblants ». À la veille de la bataille, quand le roi conquérant veut élever le moral de ses troupes, il demande à son chapelain de vanter l'excellence de sa magie et même de sa sorcellerie (abhicâra), et tout aussitôt ce sont les yantrika, "machinistes", spécialistes des différents yantra, suivis des astrologues, qui sont invités à faire l'éloge de leur propre savoir-faire, tellement supérieur à celui de l'adversaire.

Voici, avec quelques commentaires explicatifs, des extraits de ce que nous lisons en Arthaçâstra XIII 1 et 2 :

1. Le roi conquérant qui veut s'emparer d'une place forte de l'ennemi doit soulever d'enthousiasme son propre camp et emplir de terreur le camp adverse, en faisant répandre le bruit qu'il est omniscient et qu'il est en accord avec les divinités. Pour ce qui est de l'omniscience, voici comment il doit procéder : il obtient des informations secrètes sur ce qui se passe dans les maisons et les communique aux chefs de ces familles [il s'agit de leur montrer que rien de ce qui se passe chez eux n'échappe à la connaissance du roi conquérant, ni les faits qu'ils ignoraient eux-mêmes, ni ceux qu'ils pensaient pouvoir garder secrets]. Il produit en public des individus que des agents spécialisés dans l'élimination des épines ont découverts et les dénonce comme des ennemis du roi ${ }^{8}$.

Il dit par avance ce que sera une requête ou un présent qu'on s'apprête à lui faire : c'est qu'il en a pris connaissance par des signes imperceptibles pour autrui que lui font des agents qui savent ce qu'il en est, ou par des moyens qui relèvent de la «science de l'association " [on appelle ainsi les arts d'agrément : chanter, danser, tenir son rôle dans la conversation, amuser ou intéresser son entourage et s'en faire aimer, jouer à des jeux qui mettent en œuvre mimiques et signes convenus]. Il montre aussi qu'il est au fait d'événements qui viennent de se produire, ce jour même, en pays lointain : c'est qu'il les a appris par un pigeon apprivoisé porteur d'un message scellé.

Pour persuader les populations qu'il est en accord avec les dieux, il se laisse voir dans un sanctuaire consacré au dieu du feu en train de converser, dans une attitude d'adoration rituelle, avec des divinités : en fait des hommes à lui, empruntant des passages souterrains, se sont glissés dans les statues creuses installées dans ces sanctuaires. Ou encore il parle, dans une attitude d'adoration rituelle, à des agents à son service qui apparaissent comme des dieux serpents ou comme Varuna sortant de l'eau [Varuna est le dieu des eaux, c'est aussi le dieu des serments : il châtie les parjures. Les serpents, Nâga, sont aussi des divinités aquatiques, souvent représentées avec une face humaine]. Ou bien en disposant adroitement, de nuit, un récipient plein de sable marin, il fait apparaître toute une rangée de flammes qui brûlent sous l'eau'. Ou encore, il se montre debout sur un bateau maintenu immergé par des filets chargés de pierres [et donne l'impression de marcher sur l'eau].

Ou encore : il fait surgir un homme dont la tête - sauf le nez - est masquée par une vessie ou une enveloppe embryonnaire, le nez étant enduit [de façon à être lumineux 

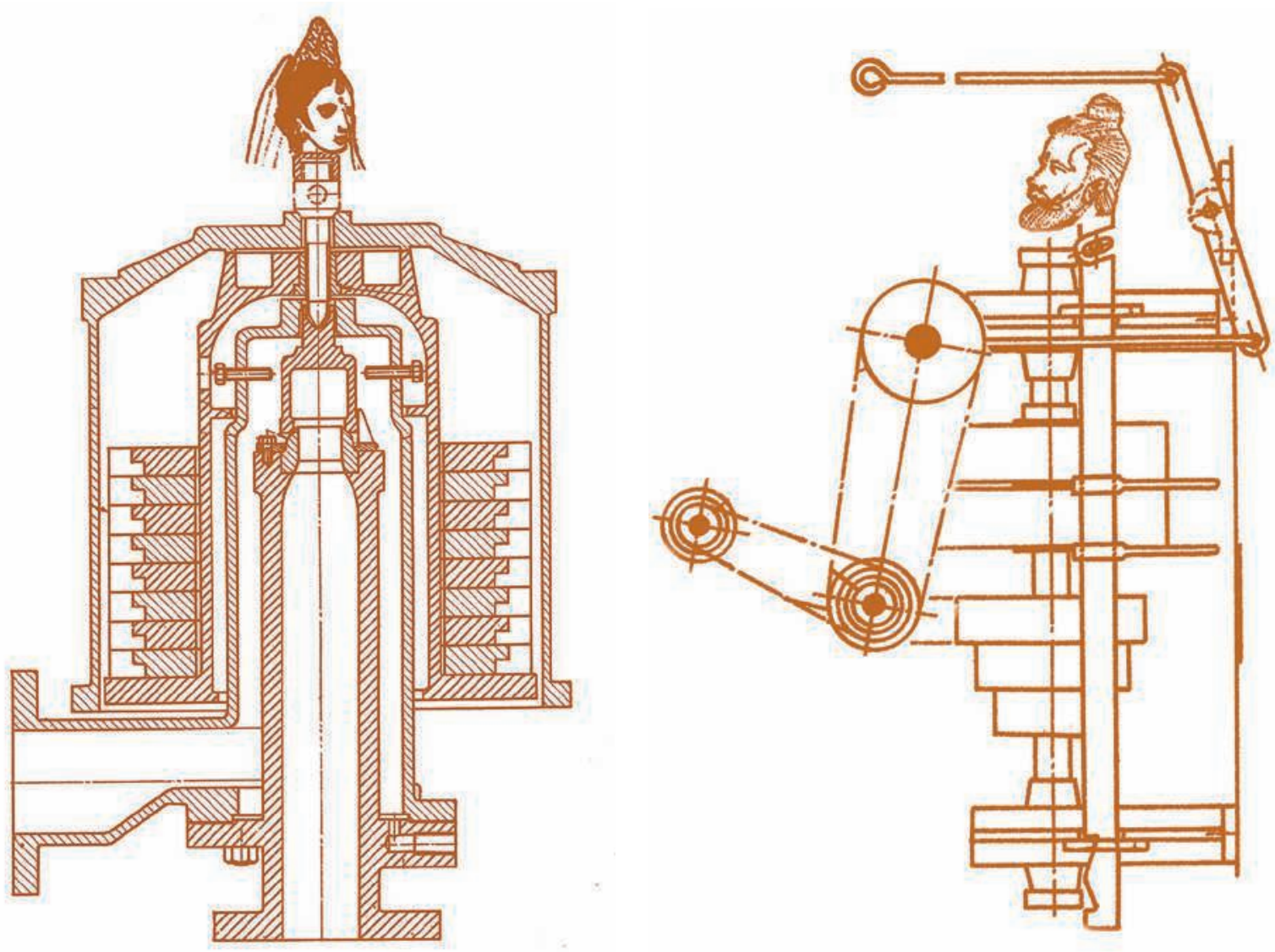

Fig. 6 Mécanismes d'automates indiens. Dessins Emmanuel Grimaud.

et brillant] d'une huile qu'on a mise à bouillir cent fois avec des entrailles de daim moucheté ou de la graisse de crabe, de crocodile, de dauphin ou de loutre : tel est l'aspect des créatures démoniaques qui rôdent en troupe la nuit. [Le nez est brillant et comme illuminé, alors que le reste du visage est noyé dans l'ombre. Réduits à ces taches de lumière mobiles, les agents du roi conquérant apparaissent aux spectateurs comme des figures démoniaques, suscitées et mises en mouvement à sa guise.] Voilà pour ce qui est des mouvements dans l'eau. On se sert de ces personnages pour faire entendre des paroles qui sont censées être prononcées par Varuna ou par les jeunes filles Nâga. Et le roi a des conversations avec eux. Quand ils sont en colère, leur bouche crache le feu et la fumée.

Devins, interprètes de présages, astrologues ${ }^{10}$, bardes spécialisés dans la récitation des Purâna $a^{11}$, voyants, agents secrets, tous ceux qui ont participé à ces stratagèmes ou qui en ont été témoins doivent faire connaître dans le territoire du roi conquérant ce pouvoir qu'il a de s'entretenir avec des personnages surnaturels. Et en territoire ennemi ils doivent aussi parler de ces rencontres face à face avec les dieux et de la façon dont il a acquis, d'une source divine, son armée et son trésor. Et dans les questions posées aux dieux, dans le vol des corbeaux, dans les signes qui relèvent de la "science du corps " [technique de divination : le devin touche le corps de celui qui lui pose des questions et règle sa réponse sur ce que ce que ce contact lui aura appris], dans les rêves, dans ce que disent les animaux et les oiseaux, ils doivent trouver des indices pour prédire la victoire du roi conquérant et l'inverse pour l'ennemi. Et aussi ils doivent marquer par un battement de tambour le passage d'un météore [fauxsemblant produit par un procédé enseigné en XIV 2,30] dans la constellation de [naissance de] l'adversaire...
-

10. Les astrologues jouent un rôle immense dans la vie des Indiens : nulle décision de quelque importance ne se prend sans l'avis de ces experts. Curieusement, le roi conquérant, s'il doit tenir compte de leur importance sociale et s'il a, dans son entourage et même dans son administration, un grand nombre de devins, d'interprètes des signes et donc d'astrologues, reçoit cependant de l'auteur de l'Arthaçâstra ce ferme conseil (IX 4, 25-26) : « L'esprit faible qui ne cesse de consulter les étoiles : c'est le but à atteindre qui est luimême la constellation favorable pour y parvenir. Que peuvent ici les étoiles?»

11. Les Purâna [《Antiquités 》) sont les livres sacrés de l'hindouisme après la période védique. Ils consistent principalement en longs récits de mythes propres à telle ou telle divinité, mais incluent des développements très divers sur le rituel, la cosmogonie, l'ordre social, les doctrines philosophiques, I es sciences profanes. 


\section{-}

12. Une forme classique de la révolte (kopa) des sujets est l'émigration en masse : le peuple part à la recherche d'une autre terre, d'un autre roi.

13. Les différents styles de vie ascétique se marquent par des différences dans la manière de se vêtir et aussi de traiter sa chevelure : aux tondus et aux nattés seuls mentionnés ici, il faut ajouter la catégorie plus farouche des hirsutes.

14. L'ascète, ici, ne se contente pas de se laisser voir par le roi. II veut aussi le rendre témoin de son entrée dans les flammes, de sa mort et de sa résurrection. Il lui manifeste sa faveur 1) en lui donnant la possibilité de formuler trois vœux, 2) en le chargeant d'organiser une grande fête, avec des spectacles, à cette occasion.

15. Un « trésor en fleurs » est un trésor qui ne reste pas tel quel mais continue à croître dans sa cache souterraine, à la façon dont croît un végétal. Cette mise en scène compliquée tend à montrer que l'ascète a trouvé la trace d'un chemin de fourmis devant le conduire à un trésor gardé par un cobra et qu'il est prêt à faire profiter le roi de sa trouvaille.

Le but ici, comme dans les situations précédentes, est de capter l'attention du roi et de l'attirer dans un lieu fixe, où il devra être seul et où on pourra facilement l'assassiner.

16. Magie noire : le pseudo-ascète, agent au service du roi conquérant, persuade le roi adverse de venir regarder une image de son ennemi et, par des incantations et gestes magiques appropriés effectués sur cette image, de provoquer la mort de ce dernier. Le but, rappelons-le, est de créer une situation où le roi adverse est isolé, sans protection, absorbé dans ce qu'il fait ou ce qu'il voit : conditions favorables pour le mettre à mort. Accessoirement, la mort, par assassinat, du roi adverse pourra être imputée à l'action en retour exercée par l'effigie du roi conquérant, si grande est sa puissance!

17. Au cours des cérémonies organisées pour obtempérer à ces ordres, les agents du roi conquérant trouveront le moyen d'isoler le roi adverse.
Quand il y a des famines [chez l'adversaire], des troubles causés par des brigands ou des tribus de la forêt, les agents du roi conquérant doivent inciter les habitants des villes et des campagnes à dire: "Demandons de l'aide à notre roi. S'il ne nous aide pas, allons ailleurs ${ }^{12}$.»

Quand ils ont dit " oui » au roi conquérant, il faut les aider en leur donnant de l'argent et des céréales, c'est là le grand prodige de l'instigation secrète à changer de camp.

2. Un ascète au crâne rasé ou orné d'une natte ${ }^{13}$, vivant dans une grotte de la montagne, déclare qu'il est âgé de quatre cents ans. Il a des disciples nattés, en grand nombre. Avec eux, il vient s'installer aux abords de la ville forte. Ses disciples se présentent au roi [adverse] et aux ministres avec des racines et des fruits, et les invitent à venir jouir de la vue du saint homme ${ }^{14}$. Celui-ci, quand il reçoit cette visite, doit leur parler des marques distinctives des rois d'autrefois et de leurs royaumes. "Au cours de ma vie, tous les cent ans j'entre dans le feu et redeviens enfant. Et maintenant sous vos yeux, ô roi, je vais pour la quatrième fois entrer dans le feu. Il faut absolument que je vous montre combien je vous honore. Faites trois vœux. " Le roi accepte et l'ascète lui dit alors : «Vous devez rester ici, avec votre fils et votre épouse, sept nuits durant, et organiser une fête avec des spectacles. » Pendant que le roi adverse est en visite [chez l'ascète], le roi conquérant l'attaque.

Ou bien : un agent se faisant passer pour un voyant des choses souterraines - qu'il ait le crâne rasé ou orné d'une natte -, avec des disciples nattés en grand nombre autour de lui, pose sur une fourmilière un bambou enduit de sang de chèvre et de poudre d'or, en sorte que les fourmis suivent ce chemin, ou encore un tube creux en or. Alors un [autre] agent secret [du roi conquérant] vient dire au roi [adverse] : «Ce saint homme sait où se trouve enfoui un trésor en fleurs ${ }^{15}$. " Questionné par le roi, le saint homme doit acquiescer, montrer ce bambou comme indice après avoir enfoui quelques pièces de monnaie dans la terre et dire : «Ce trésor est gardé par un cobra. Pour en prendre possession, il faut rendre humblement hommage à ce cobra. » Quand le roi a donné son accord, l'ascète lui dit: «sept nuits durant... » et ainsi de suite, comme précédemment.

Ou bien : tandis qu'un agent se faisant passer pour un voyant des choses souterraines se tient de nuit en un lieu solitaire, le corps entouré de flammes, d'autres agents [du roi conquérant] amènent graduellement le roi adverse à croire en lui, et lui disent: "Ce saint homme a le pouvoir de donner la prospérité. » Tout ce que le roi viendrait à lui demander, le saint homme promet de le réaliser et dit : "sept nuits durant » et ainsi de suite, comme précédemment.

Ou bien : un agent se faisant passer pour un saint homme tente le roi [adverse] par des savoirs de magie. "Tout ce que le roi viendrait à demander... » et ainsi de suite, comme précédemment.

Ou bien : un agent se faisant passer pour un saint homme s'abrite dans le temple d'une divinité en honneur dans le pays et par les fêtes fréquentes qu'il inspire gagne les principaux personnages des éléments constitutifs du royaume et peu à peu devient plus puissant que le roi...

On bien : un agent se faisant passer pour un saint homme vit à la frontière du pays. Il propose au roi [adverse] de lui faire voir [l'image de] son ennemi. Si le roi est d'accord, l'ascète fait une effigie, adresse une invocation à cet ennemi ${ }^{16}$, attire le roi dans un lieu sans issue et le tue.

Des agents qui se font passer pour des marchands viennent avec des chevaux à vendre. Ils invitent le roi à en acheter ou même lui en font présent. Et tandis qu'il est occupé à examiner leurs marchandises ou qu'il est au milieu des chevaux, ils le tuent ou le font piétiner par des chevaux.

Ou bien : des hommes de main, juchés sur un arbre sacré aux abords de la ville, se mettent à souffler dans des jarres, au milieu de la nuit, par des roseaux ou des lianes creuses, et donnent à entendre, en paroles indistinctes : " Nous allons dévorer la chair $\mathrm{du}$ roi ou de grands personnages... Nous voulons qu'on vienne nous faire un culte d'adoration ${ }^{17}$ ! " Des agents se faisant passer pour des interprètes des signes et pour des astrologues font connaître ces paroles. 
Ou bien : des agents se faisant passer pour des Nâga, le corps couvert d'huile brûlante, font grincer en les frottant les unes contre les autres des massues de fer et des pilons dans un lac sacré ou dans une citerne et disent des paroles qui vont dans le même sens [que celles dites par les ogres].

Ou bien : des agents revêtus de peaux d'ours crachent du feu et de la fumée et, prenant l'apparence de Râkshasa, font trois fois le tour de la ville par la gauche ${ }^{18}$, et dans les moments où ils interrompent leurs aboiements et leurs cris de chacal font entendre des paroles qui vont dans le même sens.

Ou bien : dans un sanctuaire, la nuit, ils mettent le feu à une statue de divinité enduite d'huile ou enveloppée d'une couche de mica et disent des paroles qui vont dans le même sens. D'autres agents les font connaître.

Ou bien : ils font couler des flots de sang de statues de divinités vénérées. Et d'autres agents déclarent que ces écoulements du sang de la divinité seront cause de la défaite dans la bataille.

Ou bien ils font savoir que dans les nuits de pleine lune et de nouvelle lune il y a des hommes qui sont mangés debout dans un sanctuaire construit sur une partie élevée du terrain de crémation ${ }^{19}$. Un agent se faisant passer pour un Râkshasa exige l'offrande d'une vie humaine. Quiconque voulant gagner une réputation de bravoure, ou pour toute autre raison, viendrait là pour voir, doit être tué par ces agents à coups de pilon de fer - en sorte que le bruit se répande qu'il a été tué par les Râkshasa. Des témoins de la scène informent le roi de ces événements surnaturels. Alors d'autres agents, se faisant passer pour devins et astrologues, prescrivent des rites de réparation pour apaiser les Râkshasa, ajoutant que, si ces rites ne sont pas exécutés, un grand malheur s'abattra sur le roi et le pays. Quand le roi a donné son accord pour ces cérémonies, ces agents [du roi conquérant] disent: «Pendant ces cérémonies, le roi, sept jours durant, doit faire lui-même des oblations quotidiennes en récitant des formules sacrées. " Puis comme précédemment.

Ou bien le roi conquérant s'expose lui-même à ces manifestations démoniaques [yoga] et fait voir qu'il y résiste victorieusement [par les rites prescrits] - de façon à édifier les ennemis [en les persuadant de l'efficacité de ces mesures]. Puis il met en œuvre ces machinations [contre ses ennemis ${ }^{20}$ ]...

Si le roi adverse fait des visites fréquentes à de saints hommes, à des ermites, à des images divines dans des sanctuaires et des stûpa [lieux de culte bouddhistes], des hommes de main [du roi conquérant] postés dans des caches souterraines ou dans des murs creux se jettent sur lui et le tuent.

Dans les lieux où le roi [adverse] en personne assiste à des spectacles, participe joyeusement à des fêtes, à des excursions, s'adonne à des jeux dans l'eau, chaque fois qu'il est amené à faire des remontrances, à prendre part à des sacrifices et des cérémonies, lors des rites de naissance ou de funérailles, quand c'est le temps d'aimer, de souffrir ou de craindre, quand son attention se relâche et que, plein de confiance, il va et vient parmi les siens, quand il se déplace sans escorte, un jour de pluie, dans la foule, quand il y a un incendie, quand il est dans un lieu désert, il faut que des hommes de main [du roi conquérant] arrivent, chargés de paquets de vêtements, d'ornements, de guirlandes, de lits et de sièges, de récipients contenant boissons fortes et choses à manger, d'instruments de musique [tous objets qui rendent leur présence vraisemblable], et que, se joignant aux agents qui étaient déjà postés là auparavant, ils se jettent sur lui et le tuent.

De ces passages de l'Arthaçâstra, on peut conclure que le roi est un esprit fort. Il sait que les hommes sont crédules, qu'ils sont prêts à accepter le surnaturel. Il joue de leur propension à accepter les prodiges et ne craint pas d'indisposer les dieux par ses manipulations qui, pour un esprit religieux, sont en quelque manière sacrilèges. Il ne met pas en question leur existence mais il affirme avec force que dans ce qui peut affecter les hommes il y a ce qui relève du divin et qui est chance ou malchance, et ce qui relève de l'action des hommes eux-mêmes, qui est bonne ou mauvaise politique. Or, ajoute-t-il, ce qui relève des dieux ou
- -

18. Quand on veut marquer sa dévotion à un sanctuaire ou une image divine, on tourne autour de cet objet dans le sens des aiguilles d'une montre, c'est-à-dire en l'ayant constamment à sa droite. Le mouvement inverse est au contraire hostile et menaçant (ou lié en quelque manière à la mort ] et caractérise les ogres et les démons (Râkshasa).

19. «lls sont mangés debout », c'est-à-dire dévorés vivants. Les terrains de crémation, encombrés de restes mal consumés, hantés par les fantômes de ceux qui n'ont pas reçu les rites funéraires convenables et par toutes sortes de forces malfaisantes, sont des lieux sinistres. Dans les contes et les pièces de théâtre, ils sont le lieu de scènes terrifiantes.

20. Le roi conquérant est suprêmement manipulateur. Il ne lui suffit pas de créer des manifestations démoniaques fictives auxquelles lui-même ne croit pas mais dont il est sûr que son adversaire les tiendra pour vraies, il fait mine aussi d'avoir été lui-même attaqué par ces démons et de leur avoir victorieusement résisté grâce à des rites que les agents à son service recommandent au roi adverse. 


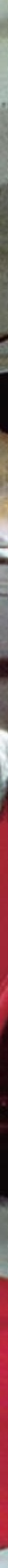




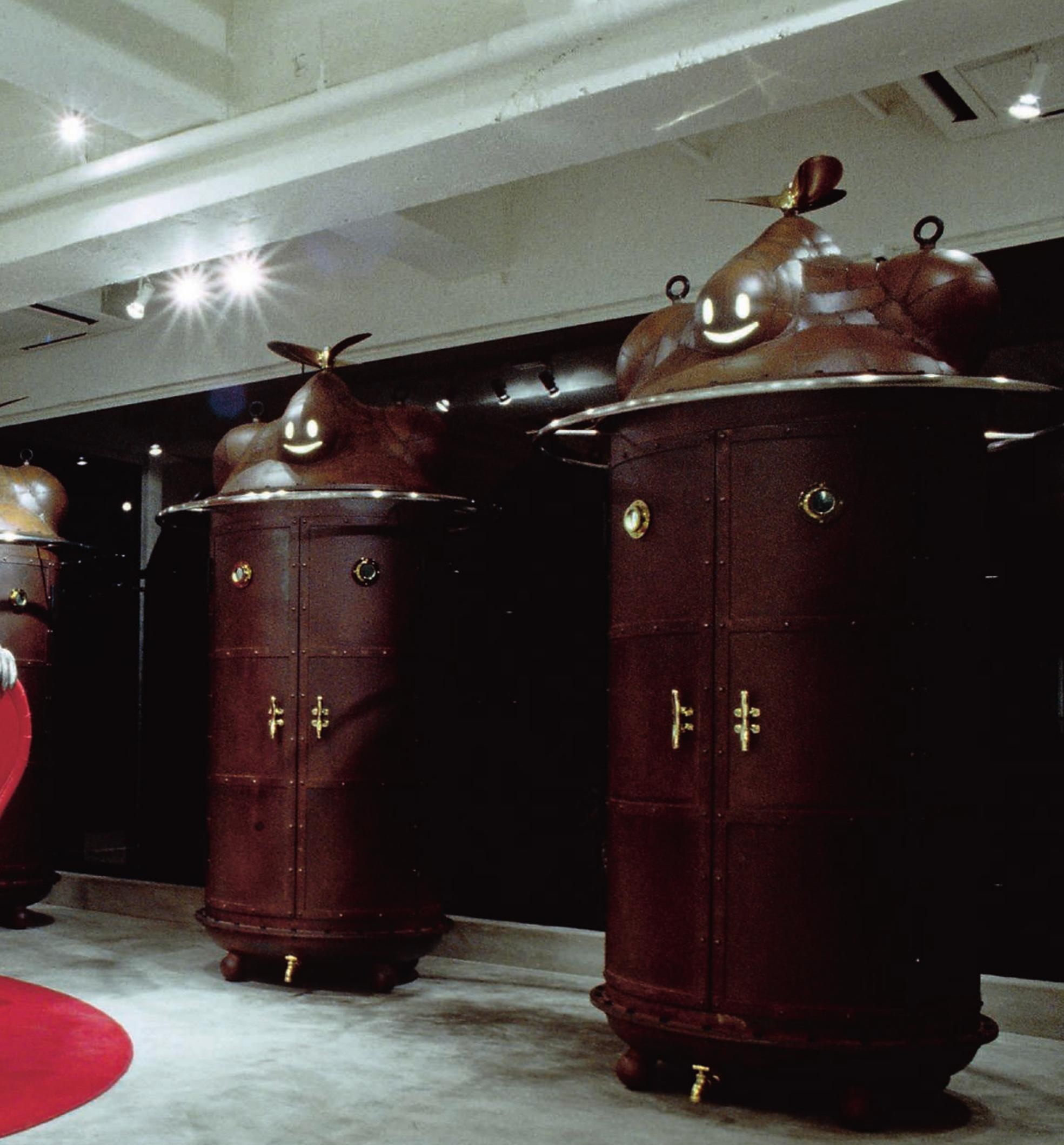


Fig. 7 (page précédente) Kenji Yanobe, Queen Mamma, 2002. Photo Seiji Toyonaga. Avec l'aimable autorisation de la Yamamoto Gendai Gallery. de la chance est impensable (VI 2, 6-12). Aussi bien il est absurde de chercher à prévoir l'avenir, on perd son temps à consulter les présages et à demander aux astres des indications sur ce qui va arriver ou sur ce qu'il faut faire. Cependant, le roi doit gouverner les hommes tels qu'ils sont, maintenir la société telle qu'elle est. Il doit tenir compte de leurs croyances et même, comme on l'a vu, en tirer parti. S'agissant des aspects «magiques» de la religion commune, et en dehors des scénarios, machines et machinations qui sont des procédés de sa politique, il s'en remet prudemment aux brahmanes spécialisés, connaisseurs de la partie "magique » du Veda, l'Atharva-Veda. Il n'est en rien un réformateur et c'est tout naturellement que dans les territoires qu'il conquiert il installe des colonies de brahmanes afin d'y faire régner le dharma, c'est-à-dire l'ordre social indissociable de l'ordre cosmique selon les principes du brahmanisme. Avec cette adjonction, qui est capitale : il prévoit des mesures d'ordre public pour que les adeptes des religions autres que le brahmanisme (il s'agit, semblet-il, des bouddhistes et peut-être des jaïns) soient respectés et que leur coexistence avec le reste de la population soit assurée; de même, les «peuples de la forêt » font partie du paysage politique, il s'agit de les gagner à soi, non de les éliminer ou de les transformer. Toutes ces données pourraient former un tableau cohérent si d'autres parties du texte ne venaient tout mettre en question: le livre XIII, d'où sont tirés les passages qu'on vient de traduire et de commenter, est en effet suivi d'un livre XIV tout entier consacré aux procédés proprement magiques auxquels le roi doit recourir pour se défendre des attaques de ses adversaires, et même pour acquérir des pouvoirs prodigieux (se rendre invisible, résister indéfiniment à la fatigue). En somme, l'esprit fort a beau être certain que la magie n'est efficace que sur les esprits qui prennent de faux-semblants pour la réalité, il a beau être lui-même dans la posture du manipulateur, il y a dans les recettes destinées à produire des effets surnaturels une forme de savoir que, dans sa volonté de puissance, il ne peut pas ne pas vouloir posséder.

École pratique des hautes études, Ve section, Centre d'études de l'Inde et de l'Asie du Sud charles.malamoud@orange.fr

mots clés / keywords: Inde ancienne // ancient India - machines // machines • automates // automata • arts de l'illusionniste // the illusionist's art • guerre psychologique // psychological warfare. 


\section{Bibliographie}

\section{Références}

FEzAs, Jean (trad.)

1997 in Somadeva, Océan des rivières de contes, édition publiée sous la direction de Nalini Balbir. Paris, Gallimard

[«Bibliothèque de la Pléiade»].

Hulin, Michel et SEnART, Émile (trad.)

2010 La Bhagavad-Gîtâ, suivie du commentaire de Sankara (extraits). Paris, Seuil [《Points »).

Porcher, Marie-Claude (trad.) 1995 Dandin, Histoire des dix princes. Paris, Gallimard («Connaissance de l'Orient 》): 104-107.

\section{RENOU, Louis}

1954 Vocabulaire du rituel védique. Paris, Klincksieck.

1966 Études védiques et pâninéennes, t. XV. Paris, De Boccard.

\section{Bibliographie succincte}

\section{Dambuyant, Marinette \\ 1971 L'Arthaçâstra, le traité politique de l'Inde ancienne, extraits choisis. Paris, Rivière.}

\section{Kangle, R.P.}

1960 The Kautilîya Arthaçâstra, t. I : A critical edition with a glossary. Bombay, University of Bombay.

1963 The Kautilîya Arthaçâstra, t. II : An English translation with critical and explanatory notes. Bombay, University of Bombay.

1965 The Kautilîya Arthaçâstra, t. III : A study. Bombay, University of Bombay.

\section{MeYer, Johann-Jakob}

1926 Das altindische Buch Von Welt-und Staatsleben. Das Arthaçâstra des Kautilya. Leipzig, Otto Harrassowitz; réimpr. Graz, Akademische Druck und Verlagsanstalt, 1977.

\section{ReNOU, Louis}

1966 «Politique et économie dans l'Inde ancienne », Journal des savants : 28-40; rééd. in Louis Renou, L'Inde fondamentale, études d'indianisme réunies et présentées par Charles Malamoud. Paris, Hermann, 1978 : 185-195.

\section{SCHARFE, Hartmut}

1968 Untersuchungen zur Staatsrechtslehre des Kautalya. Wiesbaden, Otto Harrassowitz; version anglaise augmentée : Investigations in Kautalya's manual of political science. Wiesbaden, Harrassowitz, 1993.

\section{Résumé / Abstract}

Charles Malamoud, Machines, magie, miracles - Le terme sanscrit yantra signifie «machine ». Étymologiquement, le yantra est un instrument pour contenir, capter, diriger en la contrôlant telle ou telle forme d'énergie. Une simple barrière peut être conçue comme un yantra. De même des armes comme l'arc et les catapultes, et des dispositifs tels que la noria. Par extension, ces machines mentales que sont les diagrammes grâce auxquels nous pouvons canaliser et diriger notre attention ou notre imagination sont aussi des yantra. Très tôt on a associé le yantra à la magie et aux arts de l'illusionniste. Jouer de la crédulité du public, lui faire prendre pour des réalités ce qui est le produit d'un truquage, c'est là un des aspects de la guerre psychologique. L'Arthaçâstra, traité de la science du gouvernement, donne des listes détaillées des machines et machinations que le roi utilise pour éblouir et affoler ses adversaires.
Charles Malamoud, Machines, magic, miracles - Etymologically speaking, the yantra is an instrument used to contain, captivate or direct a form of energy by controlling it. A simple barrier may be seen as a yantra, as well as weapons like a bow, a catapult or devices such as the noria. By extension, mental machines such as diagrams whereby we may canalise or direct our attention or our imagination are also yantra. From early times, the yantra has been associated with magic and with the art of illusionism. Playing upon the credulity of the audience, making them take frauds for reality are aspects of psychological warfare. The Arthaçâstra, a treatise on statecraft, provides detailed lists of machines and machinations that a king employs to dazzle and frighten his enemies. 